\title{
Whither Goest Thou, Radiopharmaceutical Therapy?
}

$\mathbf{T}$ his issue of the Journal of Nuclear Medicine includes a report of a promising agent for the radiopharmaceutical therapy of metastatic melanoma (1). The report is provocative for several reasons: it provides a potential unmet therapeutic need in a disease with a dismal prognosis, it underscores the need for a companion diagnostic imaging biomarker to appropriately select patients for therapy, it highlights the changing spectrum of molecularly targeted therapies in diseases beyond the pale of standard cytotoxic chemotherapy, and it raises the issue of whether radiopharmaceutical therapy will ever be meaningfully used in patients with cancer. This perspective details the challenges and potential of radiopharmaceutical therapy development, particularly when associated with a companion diagnostic imaging biomarker.

\section{See page 9}

Perhaps the only radiopharmaceutical cancer therapy to be used regularly is ${ }^{131} \mathrm{I}$ in differentiated thyroid cancer (2). It is, however, distinguished by its arbitrariness-there is no outcome- or data-based consensus on whom or when to treat or even how much radioactivity to administer.

Other radiopharmaceutical therapies have fared worse. Pain palliation with $\beta^{-}$-emitting radionuclides $(3,4)$ and lymphoma radioimmunotherapy (5) are used much less frequently than they ought. Often, they are used much later in the course of disease than they would be, with consequent diminution in their efficacy, leading perhaps to a vicious cycle of ever-decreasing use.

Received Sep. 24, 2013; revision accepted Sep. 26, 2013.

For correspondence or reprints contact: Chaitanya Divgi, Columbia University, $622 \mathrm{~W}$

168th St., New York, NY 10032.

E-mail: crdivgi@columbia.edu

Published online Dec. 5, 2013.

COPYRIGHT ( 2014 by the Society of Nuclear

Medicine and Molecular Imaging, Inc.

DOI: 10.2967/jnumed.113.127837
The bleak cloud on the future of radiopharmaceutical therapy in the United States now has a silver lining, however-the Food and Drug Administration has approved ${ }^{223} \mathrm{Ra}$ for the treatment of castration-resistant prostate cancer (6). Is this the dawning of a new age?

\section{FEATURES OF TARGETED RADIOPHARMACEUTICAL THERAPY}

With the exception of the latest entrant, ${ }^{223} \mathrm{Ra}$, an $\alpha$ emitter, all approved radiopharmaceutical therapies involve use of a $\beta^{-}$emitter. In some instances $\left({ }^{131} \mathrm{I}\right.$, ${ }^{153} \mathrm{Sm}$ ) the radionuclide emits photons that permit imaging; in others, the nuclide does not emit imageable photons and a surrogate image (bone scan for ${ }^{89} \mathrm{Sr},{ }^{111} \mathrm{In}$ for ${ }^{90} \mathrm{Y}$ ) is used. Imaging is used only in a qualitative manner, to determine suitability. Rarely do we, in clinical practice, use imaging to quantify radiation-absorbed dose to the tumor (or normal tissue (7)). Although this may change with the increasing availability of quantifiable nuclides and imaging modalities, it is unlikely to become a standard part of our clinical practice. It is safe to say that imaging is used primarily to determine patient eligibility for therapy.

All approved radiopharmaceutical therapies used an imaging study performed to establish suitability of therapy. This is no longer universal - the requirement for an imaging study to evaluate satisfactory biodistribution of radiolabeled ibritumomab tiuxetan (Zevalin; Spectrum Pharmaceuticals, Inc.) in patients with follicular lymphoma is no longer essential (8); in differentiated thyroid cancer patients with rising serum biomarkers (thyroglobulin), too, ${ }^{131}$ I therapy is sometimes administered even when the diagnostic image is negative (though in this instance it is more of a last-ditch effort (9)). Most professionals engaged in radiopharmaceutical therapy will nevertheless rightfully insist that we are in the business of targeted therapy and therefore need to demonstrate targeting before therapy. The paper by Mier et al. (1) adheres to that tradition.

The paper also provides excellent justification for that tradition. Although the radiopharmaceutical targets melanin, it targets only $29 \%$ of lesions, and thus pretherapy demonstration of targeting is essential to prediction of efficacy. This use of a diagnostic biomarker to identify patients who may benefit from therapy is increasingly used in this era of molecularly targeted therapies, and examples include the use of a radioiodine scan before ${ }^{131} \mathrm{I}$ therapy, bone scan before therapy for bone metastases, and immunohistochemical assays (in vitro imaging) to determine eligibility for immunotherapy (with trastuzumab or cetuximab).

Indeed, in this era of targeted therapy, it is becoming commonplace that a companion diagnostic will accompany a therapeutic through its development, with both undergoing comparable scrutiny on the road to Food and Drug Administration approval. It is therefore safe to conclude that the successful development (i.e., leading to regulatory and market approvals) of the agent proposed by Mier et al. (1) will involve the development of a predictive (imaging) assay and the radiotherapeutic itself.

\section{TANDEM DEVELOPMENT OF COMPANION DIAGNOSTIC AND THERAPEUTIC RADIOPHARMACEUTICALS}

Development seems fraught with peril not to mention financial jeopardy. If the purpose of the companion diagnostic is to identify patients likely to respond to the therapy (positive), how will such categorization be made? How will targeting be defined? Will excellent (however that is defined) targeting to a few of the lesions visualized on morphologic imaging be adequate, or will it be necessary to demonstrate targeting to all? How will the initial efficacy trials be constructed-will they be randomized: if yes, will randomization occur before or after imaging; if yes, will randomization be only in those patients with positive imaging, or in all? Questions roll on. All questions related to clinical trials of course have associated cost implications, and those in turn affect study design. It is imperative to address as many questions as possible before any efficacy studies are undertaken, so that as many clinical trials as possible fall under the carefully controlled rubric so beloved by regulators.

Issues related to product placement also need to be considered before initiation of the efficacy assessment process (an umbrella 
that includes all phase 2 and later trials leading to product approval). In this instance, given the increasing number of targeted therapies found efficacious in melanoma, what would be the patient population to be studied, and would that vary during the process (e.g., would efficacy studies select therapy-naïve or limited-therapy patients)?

Last but certainly not least: the therapy landscape is littered with promising therapeutic radiopharmaceuticals, none with a companion diagnostic imaging study: radioiodine in thyroid cancer is a grandfathered exception; bone scan positivity is similarly a clinically accepted term. Imaging before radioimmunotherapy may be considered diagnostic (to demonstrate adequate biodistribution), but it was sacrificed at the altar of convenience, with no substantive effect on use. Radiation phobia is a term tossed about to explain the lack of use of radiopharmaceuticals, and though its causes are ill-understood, its ill-effects cause angst over the future of radiopharmaceutical therapy. In sum, radiopharmaceutical therapy development currently is akin to traversal through uncharted territory to an uncertain goal.

\section{CONCLUSION}

A crucial lesson learnt from molecular therapy in cancer is that in such therapy, identification of the target phenotype is crucial to success - this is most dramatically evident in the recent fast track approval of crizotinib in ALK-positive lung cancer (a mutation that occurs in only $2 \%-7 \%$ of such cancers). Hence, the cancer treatment community is certainly attuned to the possibility of identifying subgroups of patients who are most likely to respond, and the size of that subgroup may not be a matter of concern. A targeted radiopharmaceutical therapy that is likely to work in one or more of 5 patients with melanoma certainly appears to represent an answer to an unmet need in a lethal cancer, even given the increasing number of therapeutic agents recently approved in this disease. The purpose of this article is therefore not to decry development of this agent but rather to provide perspective on the hurdles that must be addressed to make this promising therapy a reality.

\section{Chaitanya Divgi}

Columbia University

New York, New York

\section{REFERENCES}

1. Mier M, Kratochwil C, Hassel JC, et al. Radiopharmaceutical therapy of patients with metastasized melanoma with the melanin-binding benzamide ${ }^{131}$ I-BA52. J Nucl Med. 2014;55:9-14.

2. Van Nostrand D. The benefits and risks of I-131 therapy in patients with well-differentiated thyroid cancer. Thyroid. 2009;19:1381-1391.

3. Serafini AN. Samarium Sm-153 lexidronam for the palliation of bone pain associated with metastases. Cancer. 2000;88(12 suppl):2934-2939.

4. Ben-Josef E, Shamsa F, Williams AO, Porter AT. Radiotherapeutic management of osseous metastases: a survey of current patterns of care. Int J Radiat Oncol Biol Phys. 1998;40:915-921.

5. Witzig TE, Fishkin P, Gordon LI, et al. Treatment recommendations for radioimmunotherapy in follicular lymphoma: a consensus conference report. Leuk Lymphoma. 2011;52:1188-1199.

6. Parker C, Nilsson S, Heinrich D, et al. ALSYMPCA Investigators. Alpha emitter radium-223 and survival in metastatic prostate cancer. $N$ Engl J Med. 2013;369:213-223.

7. Schwartz J, Humm JL, Divgi CR, Larson SM, O'Donoghue JA. Bone marrow dosimetry using ${ }^{124}$ I-PET. J Nucl Med. 2012;53:615-621.

8. Zevalin ${ }^{\circledR}$. www.zevalin.com. Accessed November $21,2013$.

9. Leboulleux S, El Bez I, Borget I, et al. Postradioiodine treatment whole-body scan in the era of 18-fluorodeoxyglucose positron emission tomography for differentiated thyroid carcinoma with elevated serum thyroglobulin levels. Thyroid. 2012; 22:832-838. 